\title{
State of Renal Replacement Therapy Services in Ghana
}

\author{
Sampson Antwi \\ Department of Child Health, School of Medical Sciences, Kwame Nkrumah University of Science and Technology, \\ Ghana/Department of Child Health, Komfo Anokye Teaching Hospital, Kumasi, Ghana
}

\section{Key Words}

Kidney disease in failure - Renal replacement therapy ·

Dialysis · Kidney transplantation · Affordability

\begin{abstract}
Introduction: Renal replacement therapy (RRT) in the form of dialysis and kidney transplantation is a life-saving intervention for patients with kidney disease in failure for both acute and chronic cases. Ghana is an emerging economy in West Africa with close to 27 million people. The limited data that is available indicates a significant burden of kidney disease in Ghana. I analyzed the state of RRT in Ghana in this report. Method: A situational analysis report conducted to establish the availability and type of renal replacement therapy services across the country. Information was obtained from records at dialysis centers and also by interview of staff at these centers. Results: Haemodialysis services are available in 3 public and 3 private health institutions for adults in kidney failure both acute and chronic. These centers are located in the southern half of the country leaving the northern two-thirds uncovered. National Health Insurance Scheme pays for the cost of acute dialysis for up to GHC 850 ( USD 265). However, there is no insurance cover for any aspect of chronic RRT putting huge financial constraints on families, which sometimes plunge entire extended families into serious financial crisis. Kidney transplantation is available on a
\end{abstract}

limited scale at the national capital. Children only benefit from peritoneal dialysis for acute kidney injury, thanks to the partnership with Sustainable Kidney Care Foundation. There is no rescue intervention as of now for children with end stage renal failure. Conclusion: The current state of RRT services in Ghana is inadequate and calls for serious national consideration.

(c) 2015 S. Karger AG, Basel

\section{Introduction}

Renal replacement therapy (RRT) in the form of dialysis and kidney transplantation becomes life-saving intervention for patients with kidney injury/disease in failure. For both acute kidney injury in failure (AKI:RIFLE-F) and end stage renal failure (K/DOQI stage 5), death becomes imminent from severe uraemic complications and pulmonary oedema.

Ghana is a country of approximately 27 million people. There are five adult nephrologists and two paediatric nephrologists.

The exact burden of kidney disease in the community is not known. However, among hypertensive adults in four polyclinics in Accra, Ghana, Osafo et al. [1] found a chronic kidney disease (CKD) prevalence of $46.9 \%$.

\section{KARGER 125}

(C) 2015 S. Karger AG, Base

$0253-5068 / 15 / 0393-0137 \$ 39.50 / 0$

E-Mail karger@karger.com

www.karger.com/bpu 
Among all paediatric patients admitted to a Teaching Hospital in Kumasi, Ghana, over a three-year period, Antwi [2] found the overall prevalence of kidney disease to be $4.3 \%$ (664 out of 15,371 admissions).

Despite this apparent huge burden of kidney disease in the country, the availability and affordability of life-saving renal interventions like dialysis and kidney transplantation is woefully inadequate. In this report, I analyze the state of renal replacement therapy in Ghana.

\section{Method}

This was a situational analysis report conducted by the author to establish the availability and type of renal replacement therapy services across Ghana. Information was obtained from records at dialysis centers in respective institutions and also by interviewing staff at these centers.

\section{Results}

\section{Distribution of Dialysis Services in Ghana}

Among public institutions, dialysis services are available only in three health institutions: Korle-Bu Teaching Hospital-Accra, Komfo Anokye Teaching HospitalKumasi, and Cape Coast Teaching Hospital. Accra, the capital town and Cape Coast, the former capital town, are both located on the coastal lands, while Kumasi, the second largest city, is situated in the central part of the country some 300 kilometers from the capital and 600 kilometers away from the far most city in the north.

This leaves over half of the land space in Ghana uncovered with dialysis service.

Korle-Bu Teaching Hospital (KBTH) in Accra has the largest facility for dialysis and has up to 200 patients undergoing chronic haemodialysis. Besides this facility located in the renal unit of the department of Internal medicine, the Cardio-Thoracic Unit of KBTH also offers haemodialysis services for selected cases of acute kidney injury usually related to cardiac surgery.

Both the Cape Coast and Komfo Anokye Teaching Hospitals have an average of 20 clients undergoing chronic haemodialysis.

These dialysis services are run for adult patients, 3 sessions per week per client. In some extreme circumstances related to lack of funds, some clients undergo only two sessions per week in some of the centers.

Besides these public institutions, there are also limited dialysis services in three private health institutions in the country.

\section{Type of Dialysis Services Available}

In all these centers, the type of dialysis offered is haemodialysis, which is offered for both acute and chronic kidney diseases in failure. Peritoneal dialysis is not done among the adult population.

\section{Cost and Mode of Payment of Dialysis Services}

The cost per session of haemodialysis service is GHC 200 ( USD 65). Dialysis for acute kidney injury is covered by the National Health Insurance Scheme (NHIS) with a flat fee of GHC 850 ( USD 265) for the full cost of acute dialysis. Clients whose cumulative bill for dialysis sessions exceeds this amount have to top up the difference from their pockets.

The NHIS does not cover for chronic dialysis services and patients pay from their pocket or from other sources.

\section{Transplant Services}

Kidney transplantation services are carried out on a limited basis at the Korle-Bu Teaching Hospital in Accra. Started in the year 2008 by Dr. Charlotte Osafo, the number of transplant cases in this hospital stands at seventeen.

Like chronic dialysis, the cost of transplant services is privately borne. Though there is an existing ethics committee regulating the conduct of transplantation at the $\mathrm{KBTH}$ center, there is no national transplant programme in place.

\section{The Situation with Children}

\section{History of Dialysis Services in Children}

Until late 2010-2011, no dialysis services were regularly provided for children, not even for acute kidney injury. This was due in part to the nonexistence of paediatric nephrologists in the country and also due to the lack of resources for paediatric dialysis. Older children considered big enough for easy placement of central lines benefitted sparingly from adult services for the management of acute kidney injury.

Following the return after training of two paediatric nephrologists in 2008-2009, peritoneal dialysis services became available on a limited basis for children with acute kidney injury in failure [3].

In late 2011, however, following the partnership with Sustainable Kidney Care Foundation leading to the sup- 
ply of peritoneal dialysis catheters, solutions and other consumables, dialysis services have become regularly available in two centers in Ghana for children with acute kidney injury in failure.

Komfo Anokye Teaching Hospital in Kumasi started off first in 2012 and has dialyzed 50 children with 35 (70\%) of them surviving the acute phase of the intervention. Ten (10) of the 35 were deemed to have undiagnosed CKD upon further evaluation and never fully recovered their kidney function. Of the remaining 15 children who died during the dialysis intervention, late presentation and complications of the primary condition largely accounted for these deaths.

Korle-Bu Teaching Hospital in Accra has just taken off and has so far performed 5 cases of acute peritoneal dialysis in children.

These dialysis services for children are done only for acute kidney injury.

No chronic dialysis services are offered for children though there is a huge burden of chronic kidney diseases among them [4].

\section{Challenges with Dialysis Services}

\section{Affordability}

The biggest challenge facing dialysis service in Ghana is affordability. The NHIS does not cover for chronic dialysis services. As a result, the accessibility of these services is pocket driven. Though the cost per session excluding laboratory investigations and medicines is a modest GHC 200 ( USD 65), this translates into GHC 2,400 ( USD 780) per month ( USD 9,360/year) far in excess of what the average Ghanaian can afford. Most clients on chronic dialysis are the bread winners in their families and as such there is the need to keep them alive by paying for haemodialysis sessions from the extended family finances. This has the potential of placing a huge strain on the family resources, particularly when dialysis becomes a life-long procedure with no kidney transplantation in sight. Families have suffered huge financial crises as a result of this practice. Other clients, in active employment, are supported by the industrial sector.

For children who are often considered unproductive, chronic dialysis services is not a reality.

\section{Constant Electricity Supply}

In a country where there is energy insufficiency with often power rationing christened 'dum sor dum sor' (put off put on), the reliance on constant electricity supply for haemodialysis could be a challenge. Some centers have acquired backup generators, which put extra cost on their operations.

\section{Discussion}

I have outlined in detailed the distribution and type of RRT services available in a sub-Saharan country. Though dialysis and kidney transplantation services exist, their distribution is tilted towards the southern half of the country. The major challenge to accessibility of dialysis services is affordability. With a country whose minimum wage for workers is GHC 6.00 ( USD 2.00), the current situation of out of pocket payment for chronic RRT service leaves thousands of the populace unable to afford and access these services. Though peritoneal dialysis services for AKI exist for children, the situation of chronic RRT in children, often considered unproductive, is rather sorrowful. It is necessary for all to dispassionately discuss the issue of funding for the cost of renal replacement therapy. The Ghana Kidney Association and Ghana Kidney Foundation should probably take the lead in initiating these discussions. The setting up of a national transplant programme would guarantee a sustainable transplant programme that will curtail the unavailability of chronic dialysis service as is the case in most centers in the country.

The existence of insurance cover for acute dialysis services is commendable. The programme should probably expand to cover part, if not whole, of the cost of chronic dialysis as is the practice in many countries. Such a facility could rope in children into chronic dialysis services and ultimately kidney transplantation.

\section{Conclusion}

Though some progress has been made in recent years, the current state of renal replacement therapy services in Ghana is inadequate. Funding for chronic renal replacement therapy should be explored as an urgent measure.

\section{The Way Forward/Recommendations}

Chronic peritoneal dialysis services, for example, chronic ambulatory peritoneal dialysis should be integrated into the dialysis service to offset the problem of erratic electricity supply during haemodialysis. 
There is the need to develop a national transplantation programme to facilitate large-scale kidney transplantation.

To make dialysis and kidney transplant services more accessible to the populace, a fund should be set aside managed by investment consultants to offset some of the cost of renal replacement therapy. Contributions to such a fund should be from members of the civil society, industry, and government.

The NHIS should broaden its coverage to cover some aspects of chronic renal replacement therapy.

\section{Acknowledgement}

The cooperation and support of the staff of dialysis centers across the country is hereby acknowledged.

\section{Disclosure Statement}

None to declare.

\section{References}

Osafo C, Mate-Kole M, Affram K, Adu D: Prevalence of chronic kidney disease in hypertensive patients in Ghana. Ren Fail 2011; 33:388-392.
2 Antwi S: Childhood renal disorders in Ghana: a 3-year data review; in 16th Congress of International Pediatric Nephrology Association. Shanghai, China, 2013. Book of Abstracts.

3 Antwi S: Peritoneal dialysis using improvised PD catheter and self-constituted dialysis solution. Pediatr Nephrol 2010;25:1938 (abstract).
4 Antwi S: Prevalence and Epidemiology of End Stage Renal Failure in children; in 12th Congress of African Association of Nephrology/ 5th Congress of African Paediatric Nephrology Association/2nd Congress of Ghana Kidney Association. Accra, Ghana, 2013. Book of Abstracts. 